\title{
Advantages and Disadvantages of Mikrotik Nv2 Protocol on Wireless Networks
}

\author{
Nader chahardah chericki \\ ghorbani \\ Sama Technical and \\ Vocational Training College, \\ Islamic Azad University, \\ Branch of Omidiyeh, \\ khoozestan, Iran
}

\begin{abstract}
In today's world of wireless communications, has a special importance in communicating.and Some of the things that makes this enormous growth is more affordable than other methods, portability, installation and rapid implementation, quality and no need for physical but this method also has its own problems, is still, If at this stage we ignore some security problems, lack of communication bandwidth is a major problem facing the problem of distance communication also seen higher, In this regard, the researchers also found several solutions have been proposed, some of which follow the general protocol and some specifically for Wi-Fi connection is created between a manufacturer of products, This article describes how the workings of mikrotik Nv2 Protocol.
\end{abstract}

Keywords: NV2, 802.11 , CSMA/CA, mac polling, mikrotik, polling

\section{Introduction}

Up to Today, solutions and several methods to access media including using CSMA / CA or using multiple access with collision avoidance [1], RTS/CTS [2] or techniques, such as PCF, HCF, CSMA/CRAP, MACA[3], All techniques to send information on physical media, One of the ways which researchers and manufacturers of wireless equipment introduced and It will be capable of data transfer rates up considerably compared to previous methods used is polling. [4], Inspection method can be divided into two groups, each to be associated with the nodes in the wireless topology and the two types of the :

1. Roll Call Polling

2. Hub Polling

\section{POLLING METHODS}

This section describes the Protocol nv2 and the advantages and disadvantages that defines.

\subsection{ROLL CALL POLLING}

In this method there is a central controller and A channel out of band communication that central controller visit all nodes by send a token to them though outband channel and Each station has data to send, send data through out-band channels and when transmit finished, send a finish message to central controller, If a station that is deemed not to send the information, the controller will inspect the other nodes. [5] In this method the control center with a series of packets containing information such as schedule and Header data to individual nodes within the network to visit them and ask to send data if has data to transfer and promulgate data transfer end.

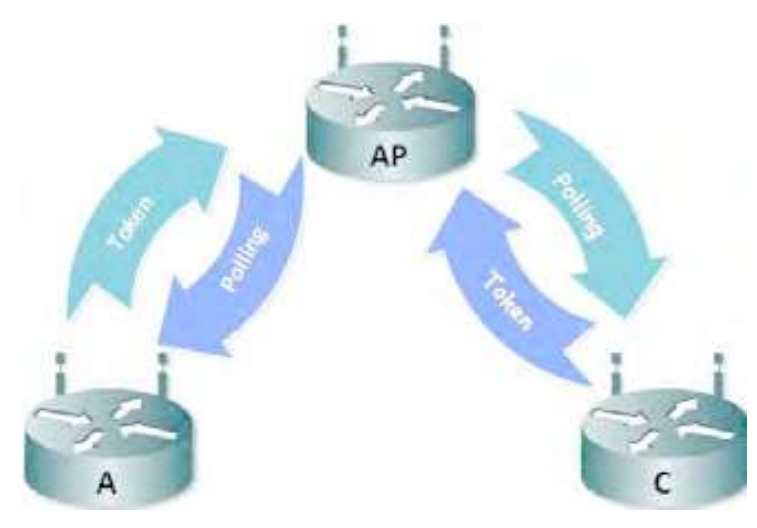

Figure 1: Roll Call Polling

\subsection{HUB POLLING}

In this method The network consists of a central station and a secondary station is zero or more. The main 
station responsible is divided into several sections, which is as follow:

1- initial value and identification network

2- Invites new stations to the network and addressing them

3- Maintain and inspect the performance of the virtual ring network.

The overall effect of this method is that the base station network has to handle and store information, Each secondary station connected to the network, such as a passive entity that expects to receive poll frame from central station, Each node is added to the network must know the address of the next stop of its own, Start the process begins when the central station give access to the media by assign poll frame to the first station of its own and If the station has data to send, start data transfer Otherwise it will send the poll frame to the next station, This too will continue to poll frames to reach the central station and the cycle starts again [6].

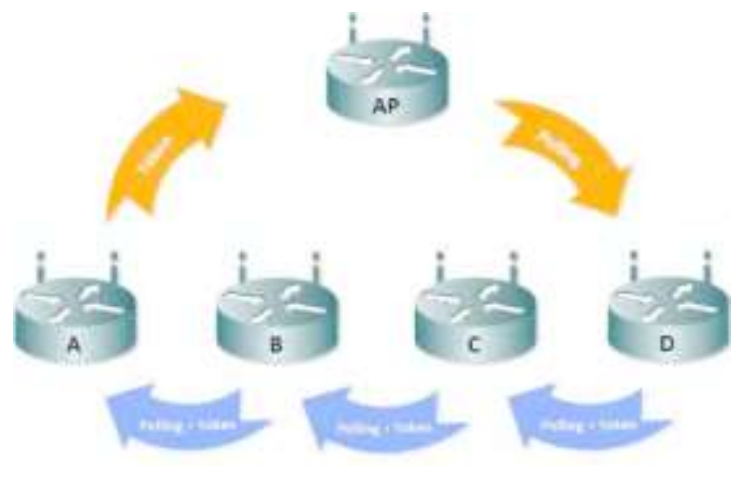

.Figure 2: Hub polling method

\subsection{POLLING BASE METHODS DISADVANTAGES}

In polling methods One of the disadvantages that reduces the time loss between the nodes of the media comes, is hands down token between stations. So that in this method, token first to be delivered to the highest station, Selected station after data transfer send token back to the central controller or an next station This problem increases the time delay between the two stations with the media which sought to reduce the amount of channels usage and Waste transfer rate yielded, However, this method can be a better alternative to using CSMA/CA, So that would be different in terms of data transfer rate of up to $05 \%$ increase, so that can increase data transfer rate up to $50 \%$ in different situation But also increases the time delay [7].

\section{Nv2 PROTOCOL}

Nv2 is one of the unique mikrotik protocol in Order to increase the rate of data transmission in wireless platform generated and Usually can be work with pci cards that has Atheros chips, this protocol use of TDMA instead of the POLLING or CSMA/CD, this method can solve hidden nodes problem in wireless communications and increase data transfer rates in Point To Point and Point To Multi Point links, Media access in Nv2 network is controlled by Nv2 Access Point. Nv2 AP divides time in fixed size "periods" which are dynamically divided in downlink (data sent from AP to clients) and uplink (data sent from clients to AP) portions, based on queue state on AP and clients. Uplink time is further divided between connected clients based on their requirements for bandwidth. At the beginning of each period AP broadcasts schedule that tells clients when they should transmit and the amount of time they can use. In order to allow new clients to connect, Nv2 AP periodically assigns uplink time for "unspecified" client - this time interval is then used by fresh client to initiate registration to AP. Then AP estimates propagation delay between AP and client and starts periodically scheduling uplink time for this client in order to complete registration and receive data from client. Nv2 implements dynamic rate selection on per-client basis and ARQ for data transmissions. This enables reliable communications across Nv2 links [8].

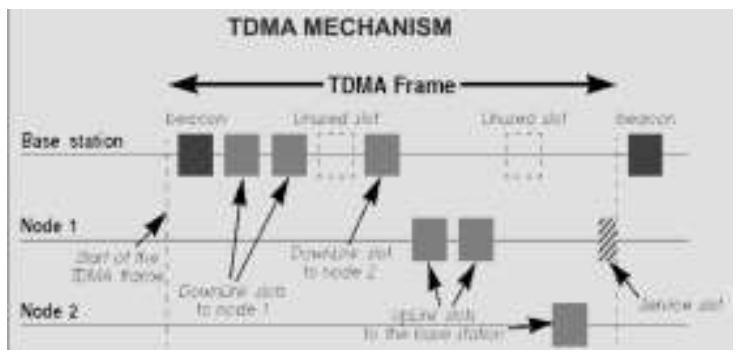

Figure 3: Nv2 structure [9].

\subsection{ADVANTAGES}
A. More Throughput
B. Lower Latency
C. Good for Point to Multipoint Networks
D. Solves Hidden Node Problem

\subsection{DISADVANTAGES}

A. Only RouterOS devices will be able to participate in Nv2 network and only RouterOS devices will see Nv2 AP when scanning.

B. Nv2 network will disturb other networks in the same channel and Nv2 network may be affected by any (Nv2 or not) other networks in the same channel. 
C. Nv2 enabled device will not connect to any other TDMA based network.

D. Does not Support $802.11 x$ standard WPA/WPA 2

Security Protocols

\section{CONCLUSION}

According to tests done on a link with a distance of about $2 \mathrm{~km}$ and Almost 150 meters height difference between send and receive points and In a warm region and dry air, The results show that due to the noise in the area and about $30{ }^{\circ} \mathrm{C}$ air temperature Nv2 protocol is better than 802.11 protocol on mikrotik products And increases the data transfer bandwidth, In addition, this protocol is secure because only mikrotik devices able to detect this protocol, Below pictures show results of the test in the winter and $30 \%$ of air humidity.

\begin{tabular}{|c|c|c|}
\hline \multicolumn{2}{|c|}{ AP Client $00: 0 C \cdot 42 \cdot E 8: 7 A: 13$ > } & $\square \times$ \\
\hline General $802.1 \mathrm{x}$ Signal & Nstreme NV2 Statistics & OK \\
\hline Last Activity: & $0.000 \mathrm{~s}$ & Remove \\
\hline$T x / R x$ Signal Strength: & $-49 /-49 \mathrm{dBm}$ & \\
\hline Tx/Rx Signal Strength Ch0: & $-53 /-53 \mathrm{dBm}$ & Reset \\
\hline Tx/Rx Signal Strength Ch1: & $-51 /-52 \mathrm{dBm}$ & Copy to Access List \\
\hline$T_{x} / R_{x}$ Signal Strength Ch2: & & Copy to Connect List \\
\hline Signal To Noise: & $67 \mathrm{~dB}$ & Ping \\
\hline$T x / R x C C Q:$ & $95 / 96 \%$ & MAC Ping \\
\hline P Throughput: & $47003 \mathrm{kbps}$ & Telnet \\
\hline
\end{tabular}

Figure. 4. 802.11 Protocol signal quality

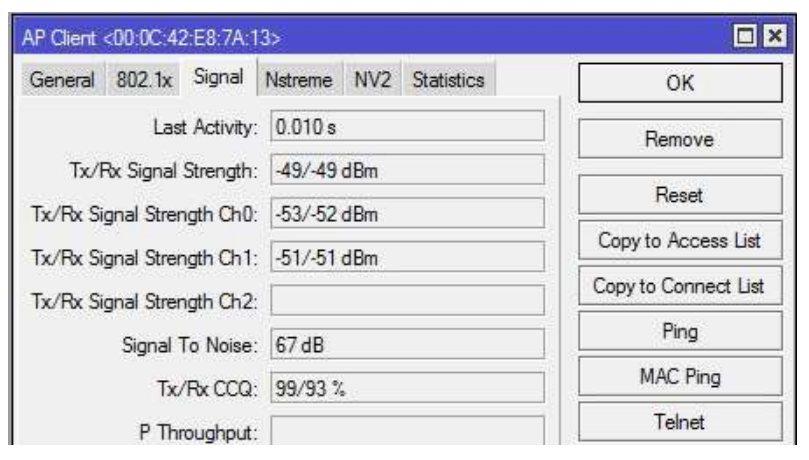

Figure. 5. Nv2 Protocol signal quality

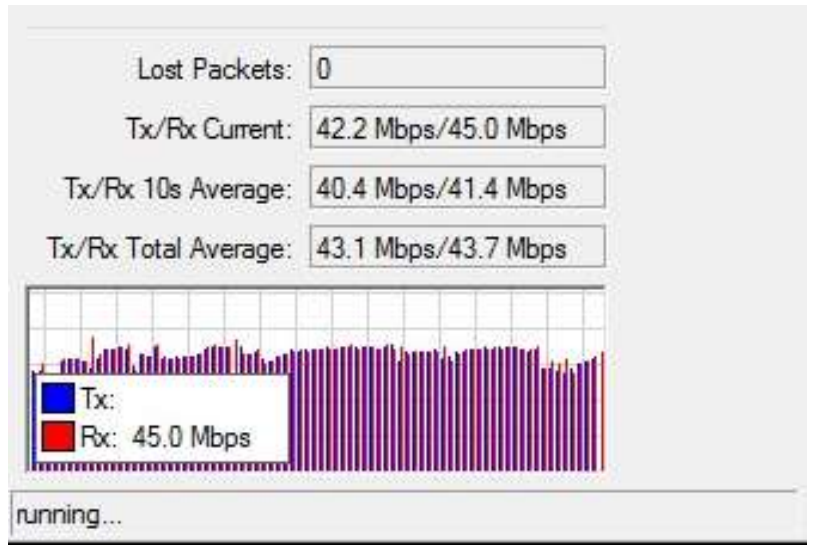

Figure. 6. Nv2Protocol TCP bandwidth test

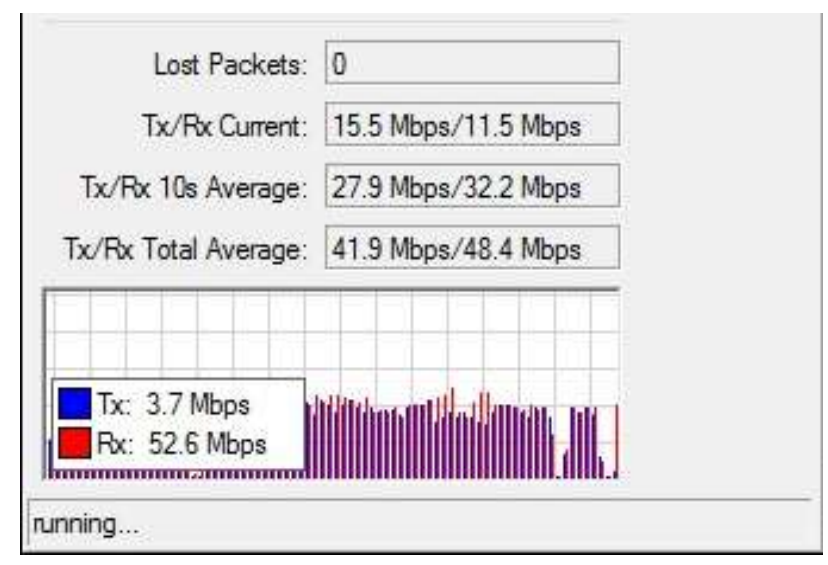

Figure.7. 802.11 Protocol TCP bandwidth test

\section{REFERENCES}

[1] andrezuguete,improved csma/ca protocol for ieee 802.11, next generation internet network 2008.Ngi 2008 IEEE 28-30 april 2008 p 247-252,krakew

[2] revisit of ris/cts exchange in high-speed IEEE 802.11 networks, World of Wireless Mobile and Multimedia Networks, 2005. WoWMoM 2005. Sixth IEEE International Symposium on a, 13-16 June 2005 ,p 240-248

[3] http://en.wikipedia.org/wiki/media_access_control

[4] 802.11 offers a polling channel access mechanism (point coordination function)in addition to the csma/ca one. http://www.hpl_hp_com/personal/jean_tourril hes/linux/linux.wireless.mac.html

[5] Vuong, S.T. , A low-cost and portable local area network for interconnecting PC's using electric power lines, Selected Areas in Communications, IEEE Journal on (Volume:7, Issue: 2 ), Feb. 1989 ,p 192-201. 
International Journal of Computer Applications Technology and Research

Volume 4- Issue 11, 789 - 792, 2015, ISSN: 2319-8656

[6] Young-Jae Kim, "Adaptive polling MAC schemes for IEEE 802.11 wireless LANs" 57th IEEE Semiannual , 2528 - 2532 vol.4, 2003Young-Jae Kim , "Adaptive polling MAC schemes for IEEE 802.11 wireless LANs" 57th IEEE Semiannual, 2528 2532 vol.4 , 2003

[7] Normandy Pour Reza, Moghadam Reza, NV2 protocol to increase the data transfer rate to replace the mutiny, sixteen Iranian Student Conference on Electrical Engineering, Islamic Azad University Kazeroon, 41-41 September 4311

[8] Nv2 protocol is proprietary wireless protocol developed by MikroTik http://wiki.mikrotik.com/wiki/Manual:Nv2

[9] soumilguptab haya2, Mum india 2012, prop Ritarymirdess protocols 